\title{
Modeling and Numerical Study of Ceramic Paste Extrusion
}

\author{
Boris GOLMAN ${ }^{1 *}$, Piotr SKRZYPACZ ${ }^{2}$, and Wittaya JULKLANG ${ }^{3}$ \\ ${ }^{1}$ School of Engineering, Nazarbayev University, 53 Kabanbay Batyr Ave., Nur-Sultan 010000, Kazakhstan \\ ${ }^{2}$ School of Science and Technology, Nazarbayev University, 53 Kabanbay Batyr Ave., Nur-Sultan 010000, Kazakhstan \\ ${ }^{3}$ School of Engineering, Suranaree University of Technology, Nakhon-Ratchasima, Thailand
}

\begin{abstract}
The extrusion processes of ceramic pastes, including 3D printing, are used for the production of high-value products. Ceramic paste extrusion is a complex process which depends on the paste rheological properties, die and extruder geometries, and operational parameters. Modeling and quantitative analysis of paste molding are important to design proper extrusion process for the production of high-value extrudates of desired strength, shape, and morphology. In this paper, the mathematical model of ram extrusion of ceramic materials is established, and the paste continuity and momentum equations for non-Newtonian fluid based on the modified Herschel-Bulkley viscous model were solved numerically. The effects of die geometry and paste feed rate on the distributions of paste velocity and pressure in the extruder and die were investigated numerically. As a result, the steeper radial profile of longitudinal velocity and higher value of longitudinal velocity were obtained in the narrow die. The pressure significantly increases in the die at a high feed rate, and the pressure profile is almost flat in the barrel. The rate of increase of the maximum pressure decreases with an increase of paste feed rate. The pressure steeply increases in the die of small diameter. The maximum pressure linearly increases with the ratio of die length to diameter.
\end{abstract}

\section{Introduction}

The extrusion process of ceramic pastes is commonly used for the production of high-value products, e.g., catalyst pellets for the chemical reactor (Devyatkov et al., 2016), honeycomb catalyst for purifying gas exhausted from an automobile (Govender and Friedrich, 2017), and ceramic packings for adsorption and direct heat transfer (Darakchiev et al., 2016). Recently, extrusion-based 3D printing was successfully applied for manufacturing of ceramic superconductors, zeolite monoliths, and porous scaffolds (Chen et al., 2019; Coucke et al., 2018; Vaezi et al., 2018). Ceramic paste extrusion is a complex process which depends on the paste rheological properties, die and extruder geometries, and operational parameters (Benbow and Bridgwater, 1993). The paste rheological properties are controlled by several factors including volume fraction of particles and their size distribution and shape, packing density and surface characteristics, as well as amount and properties of binder and other additives (Powell et al., 2013). Modeling and quantitative analysis of paste extrusion are important to design properly the extrusion process for the production of high-value extrudates of desired strength, shape, and morphology.

The objective of this paper is to establish appropriate mathematical model of ram extrusion of ceramic materials and use it for the optimization of the extrusion process. The paste extrusion will be modeled as a flow of non-Newtonian fluid. The paste continuity and momentum equations will be solved numerically using the modified Herschel-Bulkley viscous model. The effects of die geometry and paste feed rate on the distributions of paste velocity and pressure in the extruder and die will be investigated numerically.

\section{Model Equations}

\subsection{Incompressible Navier-Stokes equations for non-Newtonian fluid}

The modified Navier-Stokes equations for incompressible non-Newtonian fluid are formulated in the material domain $\Omega \subset \mathbf{R}^{d}, \mathrm{~d}=2,3$, and in the time interval as (Roubicek, 2009):

$$
\begin{aligned}
& \rho \frac{\partial \mathbf{v}}{\partial t}-\operatorname{div}(\boldsymbol{\tau}(\mathrm{D} \mathbf{v}, \vartheta)-\rho \mathbf{v} \otimes \mathbf{v})+\nabla p=\mathbf{f}, \\
& \operatorname{div}(\mathbf{v})=0,
\end{aligned}
$$

where $\mathbf{v}$ and $p$ denote the unknown velocity and pressure, respectively, $\rho$ stands for the paste density, $\mathrm{f}$ is the bulk force, and $d$ is the spatial dimension. Here, $\tau$ is the viscous part of the stress tensor, depending on the symmetrized velocity gradient $\mathrm{D} \mathbf{v}=\frac{1}{2}(\nabla \mathbf{v})^{T}+\frac{1}{2} \nabla \mathbf{v}$.

The model Eq. (1) is complemented by the initial condition

\footnotetext{
Corresponding author: boris.golman $@$ nu.edu.kz
} 


$$
\mathbf{v}(0, \cdot)=\mathbf{v}_{0} \text { in } \Omega
$$

and the no-slip boundary condition

$$
\left.\mathbf{v}\right|_{\Sigma}=0
$$

where $\sum:=(0, T) \times \partial \Omega$. Other types of boundary conditions can also be prescribed, e.g., the Neumann boundary condition on the outflow part of the domain boundary. In the isothermal case, the momentum equation reads as follows

$$
\left.\rho\left(\partial_{t} \mathbf{v}+(\mathbf{v} \cdot \nabla) \mathbf{v}\right)\right)-\nabla \cdot \boldsymbol{\sigma}=\mathbf{f} .
$$

The stress tensor can be represented as a combination of the volumetric and deviatoric stresses as

$$
\boldsymbol{\sigma}=-p \mathbf{I}+\boldsymbol{\tau},
$$

whereas the deviatoric stress $\boldsymbol{\tau}$ is given by

$$
\boldsymbol{\tau}=-p \mathbf{I}+2 \mu(\dot{\gamma}) \boldsymbol{\varepsilon}(\mathbf{v}) .
$$

Here, $\mu$ is the dynamic viscosity, which depends in general on the equivalent strain rate $\dot{\gamma}$, and $\boldsymbol{\varepsilon}$ stands for the strain tensor defined as

$$
\boldsymbol{\varepsilon}(\mathbf{v}):=\nabla^{s} \mathbf{v}=\frac{1}{2}\left(\nabla \mathbf{u}+(\nabla \mathbf{v})^{T}\right)
$$

where $\nabla^{s} \mathbf{v}$ is the symmetric part of the velocity gradient. The equivalent strain rate $\dot{\gamma}$ and the equivalent deviatoric stress $\tau$ are defined as

$$
\dot{\gamma}=(2 \boldsymbol{\varepsilon}: \boldsymbol{\varepsilon})^{1 / 2}, \quad \tau=\left(\frac{1}{2} \boldsymbol{\tau}: \boldsymbol{\tau}\right)^{1 / 2} .
$$

In the case of non-Newtonian fluid, the viscosity depends on the deformation process. To this end, various models for viscoplastic fluids have been established. In the present paper, we consider the Herschel-Bulkley model, which combines the existence of the yield stress, $\tau_{y}$, with the power law model for the viscosity (Herschel and Bulkley, 1926).

$$
\begin{array}{lll}
\mu(\dot{\gamma})=k \dot{\gamma}^{n-1}+\frac{\tau_{y}}{\dot{\gamma}} & \text { if } & \tau \geq \tau_{y} \\
\dot{\gamma}=0 & \text { if } \quad & \tau<\tau_{y},
\end{array}
$$

where $k$ is the consistency parameter and $n$ is the flow index. Thus, the deviatoric stress tensor is given by

$$
\begin{array}{lll}
\boldsymbol{\tau}=2\left(k \dot{\gamma}^{n-1}+\frac{\tau_{y}}{\dot{\gamma}}\right) \boldsymbol{\varepsilon}(\mathbf{v}) & \text { if } & \tau \geq \tau_{y} \\
\dot{\gamma}=0 & \text { if } \quad \tau<\tau_{y} .
\end{array}
$$

In the case when the rate of deformation tends to zero, there is a singularity in Eq. (6). In order to solve the model equations numerically, the regularized version of the Herschel-Bulkley model should be considered (Papanastasiou, 1987; dos Santos et al., 2011). Here, the modified version of the Herschel-Bulkley model is used as given by Li et al. (2013b):

$$
\begin{aligned}
& \mu(\dot{\gamma})=\frac{\tau_{y}}{\dot{\gamma}}+k \frac{|\dot{\gamma}|^{n-1}}{\dot{\gamma}_{c}^{n-1}} \text { if }|\dot{\gamma}| \geq \dot{\gamma}_{c}, \\
& \mu(\dot{\gamma})=\left[\frac{2 \tau_{y}}{\dot{\gamma}_{c}}+k(2-n)\right]+\left[\frac{k(n-1)}{\dot{\gamma}_{c}}-\frac{\tau_{y}}{\dot{\gamma}_{c}^{2}}\right]|\dot{\gamma}| \text { if }|\dot{\gamma}|<\dot{\gamma}_{c} .
\end{aligned}
$$

\subsection{Steady-state axisymmetric Extrusion Model Equations}

In the present study, the ram extruder is considered as an axisymmetric domain. In this case, the steady-state extrusion model equations for momentum transfer in the radial and longitudinal directions, and mass conservation are formulated as follows:

$$
\begin{aligned}
& \rho\left[u_{r} \frac{\partial u_{r}}{\partial r}+u_{z} \frac{\partial u_{z}}{\partial z}\right]=\rho f_{r}-\frac{\partial p}{\partial r}+\frac{1}{r} \frac{\partial}{\partial r}\left(r \tau_{r r}\right)-\frac{\tau_{r r}}{r}+\frac{\partial \tau_{r z}}{\partial z}, \\
& \rho\left[u_{r} \frac{\partial u_{z}}{\partial r}+u_{z} \frac{\partial u_{z}}{\partial z}\right]=\rho f_{z}-\frac{\partial p}{\partial z}+\frac{1}{r} \frac{\partial}{\partial r}\left(r \tau_{r z}\right)+\frac{\partial \tau_{z z}}{\partial z}, \\
& \frac{1}{r} \frac{\partial}{\partial r}\left(r u_{r}\right)+\frac{\partial u_{z}}{\partial z}=0,
\end{aligned}
$$

where $u_{r}$ and $u_{z}$ stand for the radial and longitudinal components of the velocity field $\mathbf{v}$, respectively. Notice that the momentum equation for the angular direction is neglected due to the assumed axisymmetry. In this case, the relations $\tau_{r z}=\mu(\dot{\gamma})$ and $\dot{\gamma}=\partial u_{z} / \partial r$ lead to the expression for $\tau_{r z}$ that is analogous to Eq. (7). The exact solutions for fully developed flow profiles, i.e., $u_{r}=0$, $\partial u_{z} / \partial z=0$, and $\partial \tau_{z z} / \partial z=0$, have been derived in (Li et al., 2013a).

\section{Results and Discussion}

In the present paper, the numerical solutions to the boundary value problem for Eq. (8) were obtained using

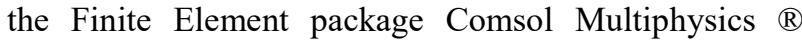
(COMSOL, 2018).

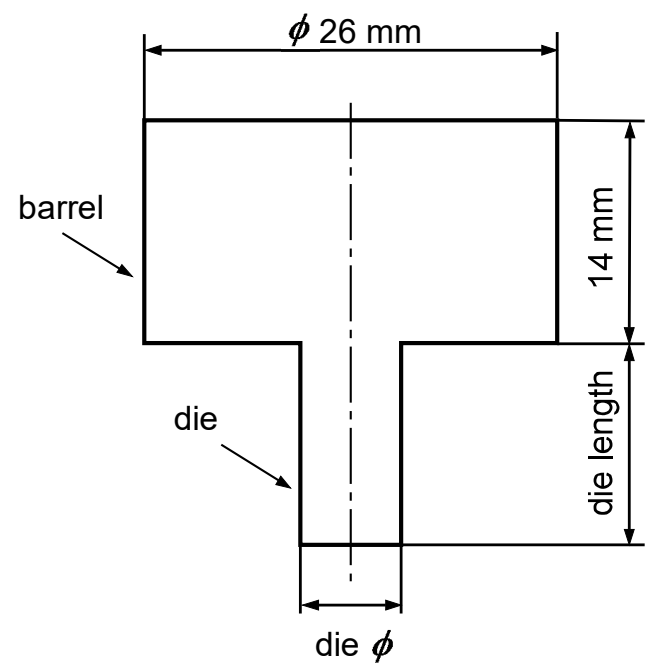

Figure 1. Illustration of ram extruder geometry. 
The extruder geometry is shown in Figure 1. To study the effect of the ratio of die length to die diameter $(L / \phi)$ on extrusion characteristics, the die diameter was fixed at $3.18 \mathrm{~mm}$ and the die length was varied as $6.36 \mathrm{~mm}$ ( $L / \phi=2), 12.72 \mathrm{~mm}(L / \phi=4), 19.08 \mathrm{~mm}(L / \phi=6)$, and $25.44 \mathrm{~mm}(L / \phi=8)$. To investigate the effect of die diameter, the dies of a fixed length of $12.7 \mathrm{~mm}$ and varied diameters of $1.59 \mathrm{~mm}, 2.19 \mathrm{~mm}, 3.18 \mathrm{~mm}$, and $6.35 \mathrm{~mm}$ were used in simulations.

The viscosity experimental data by Thomas-Vielma et al. (2008) for the paste containing $50 \mathrm{vol}$. \% of alumina (Alcoa, CT $3000, \mathrm{~d} 50=0.8 \mu \mathrm{m}$ ), 25 vol. \% of high-density polyethylene, 23 vol. $\%$ of paraffin wax, and 2 vol. \% of stearic acid were fitted with the modified HerschelBulkley model by Eq. (7). The fitted parameters are summarized in Table 1, and the fitting results are illustrated in Figure 2.

Table 1. Parameters of modified Herschel-Bulkley model

\begin{tabular}{lc}
\hline \multicolumn{1}{c}{ Parameter } & Value \\
\hline Critical shear rate, $[1 / \mathrm{s}]$ & 10.0 \\
Yield stress, $[\mathrm{Pa}]$ & 1103 \\
Consistency index, $[\mathrm{kg} / \mathrm{m} \mathrm{s}]$ & 248.7 \\
Power law index, [-] & 0.668 \\
\hline
\end{tabular}

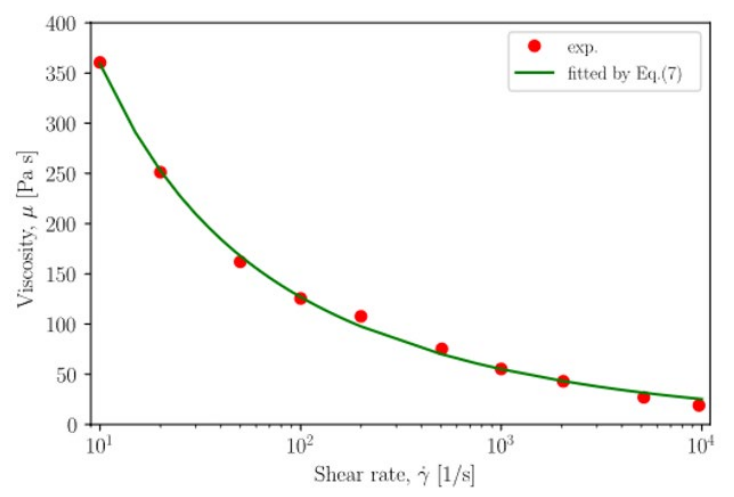

Figure 2. Fitting viscosity of alumina paste (Thomas-Vielma et al., 2008) with modified Herschel-Bulkley model

The radial distributions of the longitudinal component of the velocity, $u_{z}$, at the die end $(z=0)$ are shown in Figure 3 for dies of various diameters. The extruder feed rate was kept constant at $0.002 \mathrm{~kg} / \mathrm{s}$. The radial profile of the longitudinal velocity is steeper in a narrow die.

The axial distributions of the longitudinal velocity at the die centreline are shown in Figure 4 for dies of various diameters. The longitudinal velocity is higher in the die region of the ram extruder with narrow die due to the larger average velocity at the constant feed rate.

The axial distributions of pressure at the die centreline are illustrated in Figure 5 for various feed rates. The pressure significantly increases in the die at a high feed rate and the pressure profile is almost flat in the barrel. The variation of the maximum pressure at the upper end of a barrel with feed rate is demonstrated in Figure 6. The rate of increase in maximum pressure decreases as the feed rate increase.

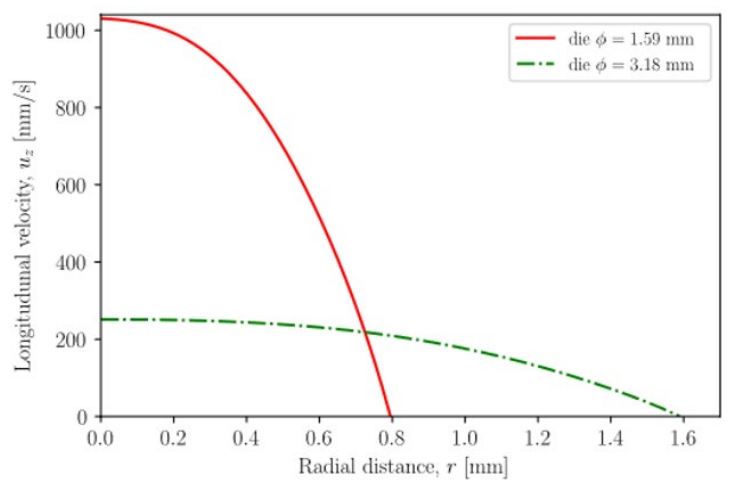

Figure 3. Radial distribution of longitudinal velocity at die end

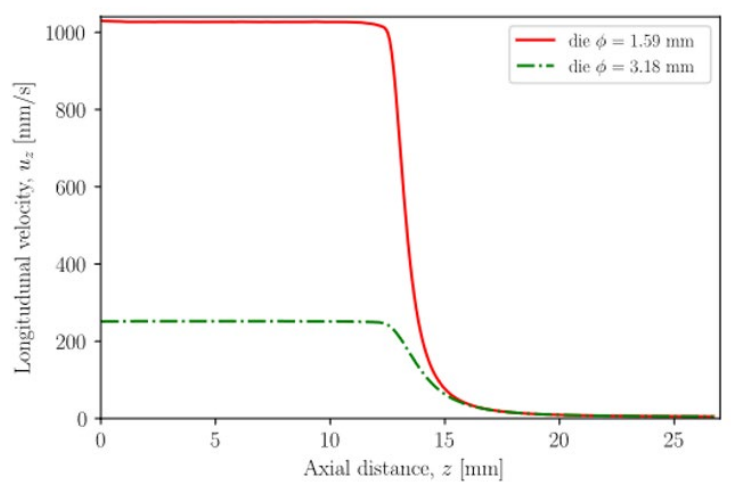

Figure 4. Axial distribution of longitudinal velocity at die centreline

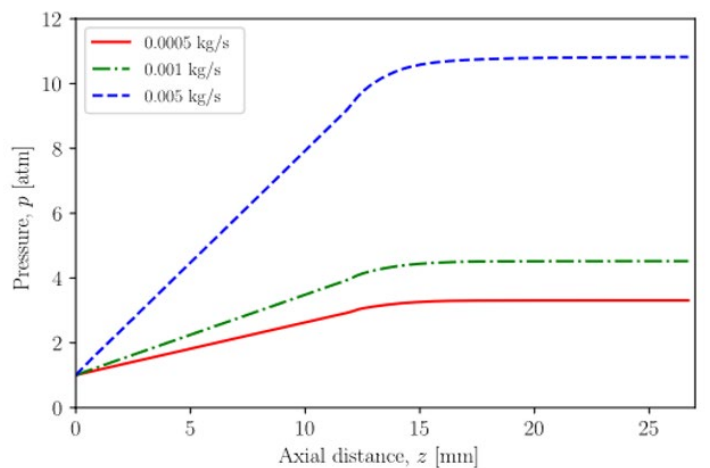

Figure 5. Axial pressure distribution at die centerline

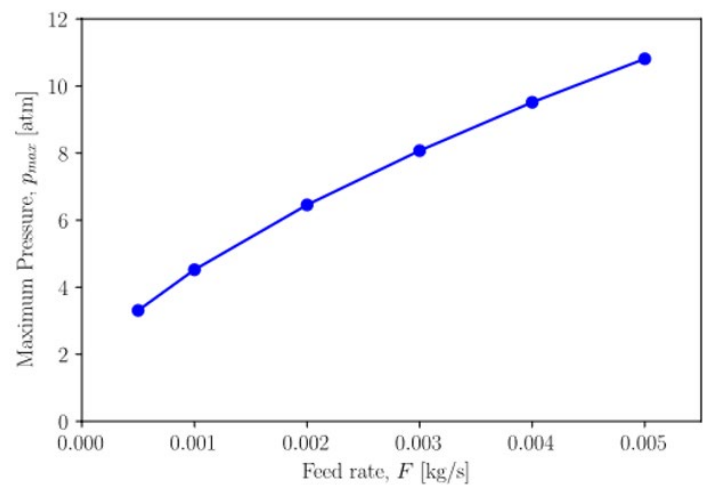

Figure 6. Effect of feed rate on maximum extrusion pressure 
The effect of die length to diameter ratio on the axial pressure distribution is shown in Fig. 7. The pressure steeply increases in the die of small diameter. The maximum pressure linearly increases with the ratio of die length to diameter, as illustrated in Fig. 8.

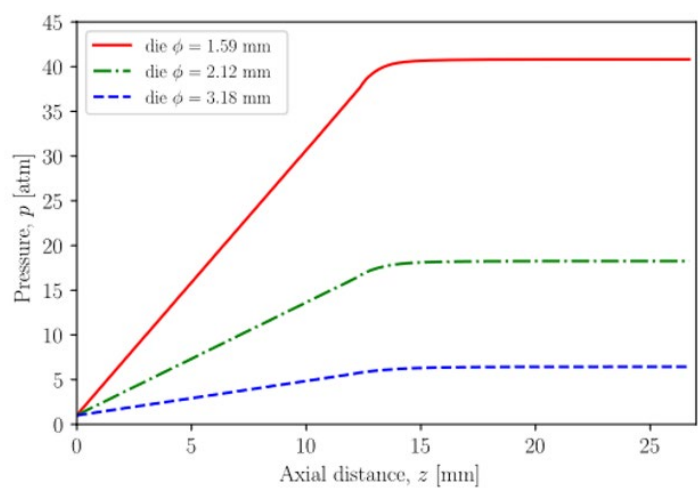

Figure 7. Axial pressure distributions at centreline of dies of various diameters.

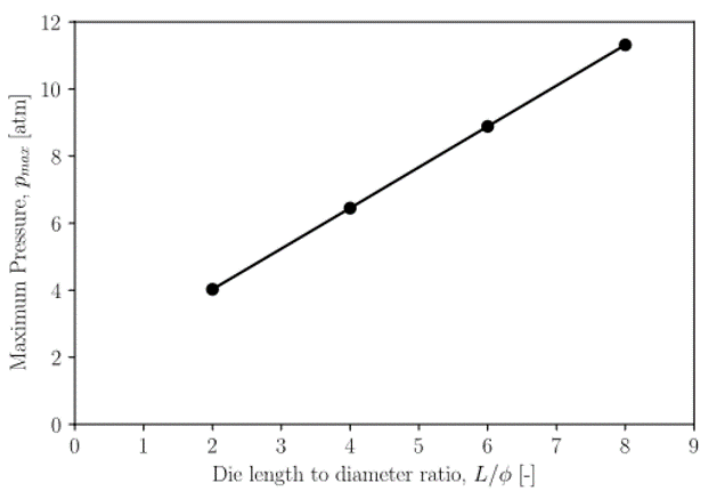

Figure 8. Effect of die length to diameter ratio on maximum extrusion pressure.

\section{Conclusions}

The extrusion of ceramic materials for the production of high-value materials was studied in the present research. The mathematical model of ram extrusion was established, and the paste continuity and momentum equations were solved numerically for non-Newtonian fluid based on the modified Herschel-Bulkley viscous model. The effects of die geometry and paste feed rate on the distributions of paste velocity and pressure in the extruder and die were investigated numerically. As a result, the steeper radial profile of longitudinal velocity and higher value of longitudinal velocity were obtained in the narrow die. The pressure significantly increases in the die at a high feed rate, and the pressure profile is almost flat in the barrel. The rate of increase in maximum pressure decreases as the paste feed rate increases. The pressure steeply increases in the die of small diameter. The maximum pressure linearly increases with the ratio of die length to its diameter.

\section{Acknowledgments}

We would like to acknowledge the contribution of Palachai Klanghiran in setting the COMSOL simulation model.

\section{References}

Benbow, J. and J. Bridgwater; Paste Flow and Extrusion, Clarendon Press, Oxford, U.K. (1993)

Chen, Z., Z. Li, J. Li, C. Liu, C. Lao, Y. Fu, C. Liu, Y. Li, P. Wang, and Y. He; "3D Printing of Ceramics: A Review," J. Eur. Ceram. Soc., 39, 661-687 (2019)

COMSOL; COMSOL Multiphysics ${ }^{\circledR} \quad$ v. 5.4., www.comsol.com, COMSOL AB, Stockholm, Sweden (2018)

Couck, S., J. Cousin-Saint-Remi, S. Van der Perre, G. V. Baron, C. Minas, P. Ruch, and J. F.M. Denayer; "3Dprinted SAPO-34 Monoliths for Gas Separation," Microporous Mesoporous Mater., 255, 185-191 (2018)

Darakchiev, R., S. Darakchiev, D. Dzhonova-Atanasova, and S. Nakov; "Ceramic Block Packing of Honeycomb Type for Absorption Processes and Direct Heat Transfer," Chem. Eng. Sci., 155, 127-140 (2016)

Devyatkov, S. Yu., A. Al. Zinnurova, A. Aho, D. Kronlund, J. Peltonen, N. V. Kuzichkin, N. V. Lisitsyn, and D. Yu. Murzin; "Shaping of Sulfated Zirconia Catalysts by Extrusion: Understanding the Role of Binders," Ind. Eng. Chem. Res., 55, 6595-6606 (2016)

dos Santos, D., S. Frey, M. Naccache, and P. de Souza Mendez; "Numerical Approximations for Flow of Viscoplastic Fluids in a Lid-driven Cavity," J. NonNewton. Fluid Mech., 166, 667-679 (2011)

Govender, S. and H. B. Friedrich, "Monoliths: A Review of the Basics, Preparation Methods and Their Relevance to Oxidation," Catalysts, 7, 62 (2017)

Herschel, W. and R. Bulkley; "Measurement of Consistency as Applied to Rubber Benzene Solutions," Proc. Am. Soc. Testing Mater., 26, 621-629 (1926)

Li, M., L. Tang, R. G. Landers, and M. C. Leu; "Extrusion Process Modeling for Aqueous-based Ceramic PastesPart 1: Constitutive Model," J. Manuf. Sci. Eng., 135, 051008 (2013a)

Li, M., L. Tang, R. G. Landers, and M. C. Leu, "Extrusion Process Modeling for Aqueous-based Ceramic PastesPart 2: Experimental Verification," J. Manuf. Sci. Eng., 135, 051009 (2013b)

Papanastasiou, T. C.; "Flows of Materials with Yield," $J$. Rheol., 31, 385-401 (1987)

Powell, J., S. Assabumrungrat, and S. Blackburn; "Design of Ceramic Paste Formulations for Co-Extrusion," Powder Technol., 245, 21-27 (2013)

Roubicek, T., "On Non-Newtonian Fluids with Energy Transfer," J. Math. Fluid Mech., 11, 110-125 (2009) 
Thomas-Vielma, P., A. Cervera, B. Levenfeld, and A. V'arez; "Production of Alumina Parts by Powder Injection Molding with a Binder System Based on High Density Polyethylene," J. Eur. Ceram. Soc., 28, 763-771 (2008)

Vaezi, M., G. Zhong, H. Kalami, and S. Yang; "Extrusion-based 3D Printing Technologies for 3D Scaffold Engineering," pp. 235-254, Functional 3D Tissue Engineering Scaffolds. Woodhead Publ., Cambridge, U.K. (2018) 